\title{
Fibrinólisis de baja dosis facilitada por ultrasonido dirigida por catéter en tromboembolismo pulmonar agudo. Reporte de un caso
}

\author{
Ultrasound-assisted catheter-directed low-dose thrombolysis for acute pulmonary \\ embolism. Case report
}

\author{
Héctor H. Escutia-Cuevas ${ }^{1 *}$, Marco A. Alcántara-Meléndez ${ }^{1}$, Paulette M. Fuentes-Espinosa ${ }^{1}$, \\ Guillermo Orozco-Guerra ${ }^{1}$, Alvaro D. Rentería-Valencia', Ronald E. Rivas-Gálvez², \\ Manuel A. Espinoza-Rueda², Robinsson J. Vásquez-Orozco ${ }^{2}$ y Hiram Vela-Vizcaíno \\ ${ }^{1}$ Servicio de Cardiología Intervencionista; ${ }^{2}$ División de Cardiología. Centro Médico Nacional 20 de Noviembre, Instituto de Seguridad y Servicios \\ Sociales de los Trabajadores del Estado (ISSSTE). Ciudad de México, México.
}

La tromboembolia pulmonar (TEP) es una de las principales causas de morbilidad y mortalidad en todo el mundo. La incidencia anual de TEP es de aproximadamente 600,000 casos, y hay de 100,000 a 180,000 muertes relacionadas, por lo que es la principal causa prevenible de muerte entre pacientes hospitalizados ${ }^{1}$. La tasa de mortalidad a 30 días de pacientes con TEP que desarrollan choque cardiogénico oscila entre el 16 y el $25 \%$, y en los pacientes que sufrieron paro cardíaco, entre el 52 y el $65 \%{ }^{2}$.

En cuanto a la clasificación clínica, se puede hacer una división entre TEP masiva o de alto riesgo y submasiva o de riesgo intermedio. En cuanto al embolismo pulmonar masivo, se caracteriza por inestabilidad hemodinámica, y es un padecimiento con alta mortalidad asociada, hasta tres veces superior en comparación con los pacientes que permanecen hemodinámicamente estables. Esta condición actualmente se define por la presencia de hipotensión arterial con presión sistólica (PAS) $<90 \mathrm{mmHg}$ durante más de 15 minutos o bien la necesidad de soporte inotrópico para mantener la PAS por encima de $90 \mathrm{mmHg}$. El embolismo pulmonar submasivo, o de riesgo intermedio, se define por PAS $>90 \mathrm{mmHg}$ con evidencia de disfunción del corazón derecho (ventrículo derecho [VD] dilatado y con una proporción de VD a ventrículo izquierdo [VI] $>0.9$ en la vista de 4 cámaras por tomografía o ecocardiograma) y biomarcadores elevados, como troponina o péptido natriurético $B$. Presentan un riesgo alto de evolución de la enfermedad si no se tratan de manera oportuna ${ }^{3}$.

La angiografía pulmonar por tomografía computarizada permite, no solo la visualización adecuada de la tromboembolia pulmonar hasta el nivel segmentario, sino también la visualización del VD como indicador de su disfunción. La ecocardiografía es una alternativa diagnóstica aceptable, aunque generalmente no proporciona un diagnóstico definitivo ni excluye la TEP, sí puede confirmar o excluir la sobrecarga y la disfunción grave del VD².

Actualmente, las escalas de estratificación a manera de scores, como el índice de gravedad del embolismo pulmonar (PESI), el score de Wells o el score de

\section{Correspondencia:}

*Héctor H. Escutia-Cuevas

Avenida Coyoacán 1617, Interior APH7

Col. Del Valle Sur, Del. Benito Juárez

Fecha de recepción: 2-10-2018

Fecha de aceptación: 4-12-2018

DOI: 10.24875/ACM.M19000041
Disponible en internet: 06-05-2019 Arch Cardiol Mex. 2019;89(3):283-287 www.archivoscardiologia.com 
Ginebra, son de gran ayuda, junto con los hallazgos hemodinámicos e imagenológicos, para clasificar al paciente según su riesgo de mortalidad ${ }^{3}$.

La estrategia de tratamiento óptimo continúa siendo un motivo de controversia, debido a que la mortalidad de la TEP es comparable con la del infarto agudo de miocardio, sin que haya sido posible alcanzar una reducción de la mortalidad en las tres décadas pasadas ${ }^{4}$. Para los pacientes con inestabilidad hemodinámica secundaria a embolismo pulmonar masivo, el tratamiento de elección continúa siendo la fibrinólisis sistémica ${ }^{5}$. En los últimos años ha surgido nueva evidencia que indica que, tanto en embolismo masivo y submasivo, la trombólisis de baja dosis dirigida por catéter y asistida por ultrasonido es un tratamiento altamente efectivo que reduce la sobrecarga del VD y disminuye la presión pulmonar y la carga de trombo, sin aumentar el riesgo de hemorragia, cuando se le aúna al tratamiento con heparina ${ }^{6-8}$.

El tratamiento dirigido por catéter (TDC) se puede realizar como una alternativa a la trombólisis cuando un paciente tiene contraindicaciones absolutas para la fibrinólisis sistémica, posee un riesgo muy alto de hemorragia mayor, como terapia adjunta cuando la trombólisis no ha podido mejorar hemodinámica, o bien como una alternativa a la cirugía si el acceso inmediato no está disponible. El objetivo del TDC es eliminar las obstrucciones trombóticas de las principales arterias pulmonares para facilitar la mecánica del VD y mejorar así la supervivencia. La tasa de éxito clínico, definida como estabilización de parámetros hemodinámicos, resolución de la hipoxia y la supervivencia hasta el alta, fue del $87 \%$.

La experiencia en nuestro país con esta modalidad de tratamiento aún es limitada, con escasos reportes previos existentes. Por este motivo, y con el afán de continuar generando experiencia en este tema, exponemos aquí un caso clínico que ejemplifica el uso ideal del TDC en un paciente con TEP submasiva.

\section{Reporte del caso}

Paciente femenina de 78 años de edad, con antecedente de hipertensión arterial sistémica de larga data, así como un evento vascular cerebral isquémico 2 años antes, sin secuelas aparentes. Ingresó en el Servicio de Urgencias de nuestro centro por un cuadro de 6 minutos de duración de disnea súbita en reposo, acompañada de dolor precordial opresivo, diaforesis y náuseas. Bioquímicamente, presentaba elevación sérica de troponina I $(130 \mathrm{pg} / \mathrm{ml})$, y electrocardiográficamente, con infradesnivel del segmento ST en la pared inferior. Al examen físico se encontró somnolienta, presión arterial 95/60 mmHg, frecuencia cardíaca 130 lpm, frecuencia respiratoria $26 \mathrm{rpm}$, con saturación por oximetría de pulso de $82 \%$ al ambiente, ingurgitación yugular grado III, tórax simétrico sin estertores ni sibilancias, extremidades sin edema. Ecocardiograma transtorácico: VI con volumen telediastólico de $43 \mathrm{ml}$, volumen telesistólico de $21 \mathrm{ml}$, fracción de expulsión del $70 \%$, sin alteraciones en la movilidad global o segmentarias en reposo; volumen auricular izquierdo de $30 \mathrm{ml} / \mathrm{m}^{2}$; válvula mitral sin regurgitación, válvula aórtica trivalva, sin gradiente transvalvular significativo, sin insuficiencia; VD dilatado, con función sistólica severamente deprimida, desplazamiento sistólico del plano del anillo tricuspídeo (TAPSE) de $12 \mathrm{~mm}$, onda $\mathrm{S}$ de $5 \mathrm{~cm} / \mathrm{s}$, fracción de acortamiento del 15\%; aurícula derecha dilatada, sin trombos en su interior; válvula tricúspide estructuralmente normal, con déficit de coaptación que genera jet concéntrico de insuficiencia moderada, vena contracta de $5 \mathrm{~mm}$, gradiente de regurgitación de $75 \mathrm{mmHg}$; se calcula una presión sistólica de la arteria pulmonar (PSAP) de $85 \mathrm{mmHg}$, proporción VD/VI de 0.98 (Fig. 1A). Se procede a realizar angiotomografía pulmonar en la que se encuentra el VD dilatado, proporción VD/VI de 0.99, así como defecto de llenado con oclusión total distal en la rama principal derecha de la arteria pulmonar (Figs. $1 \mathrm{~B}$ y C), lo que confirma el diagnostico de tromboembolismo pulmonar agudo submasivo (PESI score en clase $\mathrm{V}$, de muy alto riesgo, mortalidad asociada del $10-24 \%$ en 30 días).

Se inició anticoagulación con heparina no fraccionada (bolo intravenoso $80 \mathrm{Ul} / \mathrm{kg}$ y después infusión continua hasta alcanzar de forma rápida y mantenida la prolongación del tiempo de activación parcial de tromboplastina [aPTT] entre 1.5 y 2.5 veces el control), y debido a la existencia de contraindicaciones relativas de trombólisis se decide no realizarla. Con el objetivo de evaluar el TDC se realizó cateterismo cardíaco derecho de urgencia, con angiografía pulmonar y toma de las presiones de las cavidades derechas, y se encontró: PSAP $90 \mathrm{mmHg}$, presión media de la arteria pulmonar (PMAP) $50 \mathrm{mmHg}$, con flujo lento e imagen nebulosa en la rama principal derecha, con ausencia de flujo distal en la rama pulmonar inferior derecha (Fig. 2 A), válvula pulmonar sin gradiente al trazo de retiro hacia el tracto de salida del VD.

Durante el mismo procedimiento se colocó una sonda EKOS Endovascular System (BTG-IM, Bothell, Washington) de la siguiente manera: mediante un introductor 6 French venoso en la vena femoral derecha, se procedió a avanzar una guía hidrofílica 0.035 , a través 


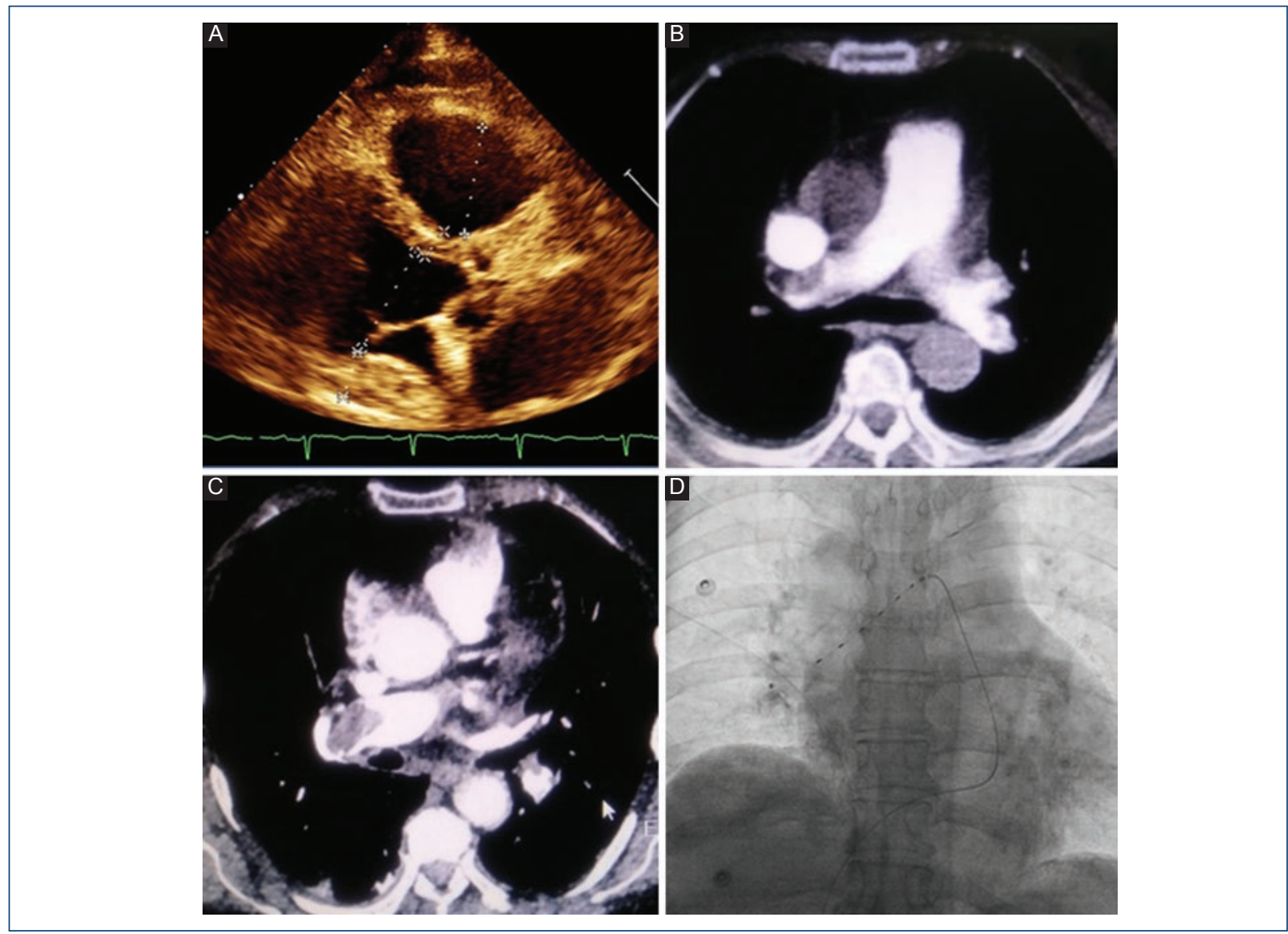

Figura 1. Fase diagnóstica de tromboembolia pulmonar y tratamiento. A: ecocardiograma transtorácico inicial, eje paraesternal largo, en el cual se observa dilatación del VD, proporción VD/VI de 0.98. B: angiotomografía pulmonar en corte axial, con defecto de llenado en la rama derecha de la arteria pulmonar que se extiende a ramas subsegmentarias. C: corte axial a nivel de T5 con mayor definición del defecto de llenado de la rama pulmonar derecha. D: resultado fluoroscópico final con sonda EKOS (BTG-IM, Bothell, Washington), colocada desde el segmento distal de la arteria lobar inferior derecha hasta la bifurcación del tronco pulmonar.

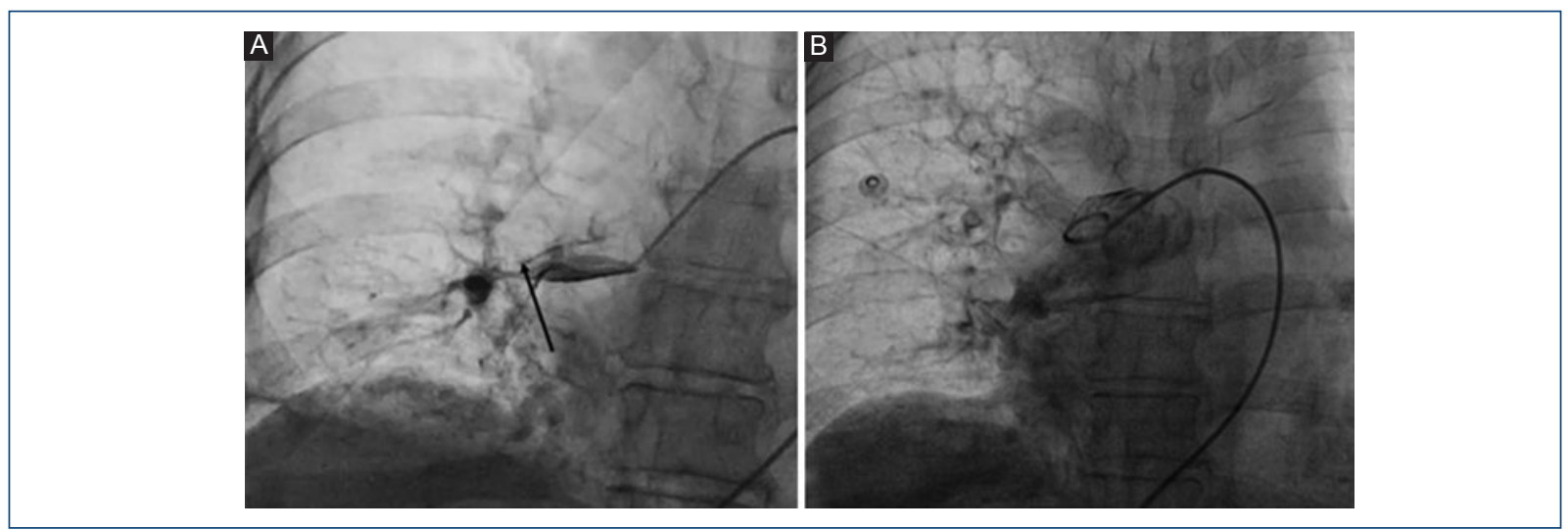

Figura 2. Angiografía pulmonar previa y posterior al tratamiento. A: en proyección anteroposterior, se observa una imagen nebulosa (flecha) en la rama principal derecha con ausencia de flujo distal en ambas ramas subsegmentarias. B: angiografía pulmonar posterior al procedimiento, que muestra mejoría del flujo distal en las ramas subsegmentarias y desaparición de la imagen sugestiva de trombo en la rama principal derecha. 
de la cual se avanzó un catéter con curva multipropósito $5 \mathrm{Fr}$ para realizar las mediciones de las cavidades anteriormente comentadas. Posteriormente, se realizó la angiografía pulmonar, ya comentada. Basándose en los hallazgos se avanzó la guía hidrofílica hacia la rama derecha y, después, a la arteria lobar inferior derecha; se hace un intercambio por la sonda EKOS y se deja colocada desde el segmento distal hasta la bifurcación del tronco pulmonar (Fig. 1 D); se corrobora el adecuado funcionamiento de la consola y la adecuada infusión de fármaco y refrigerante, dando por terminado el procedimiento, sin complicaciones.

Debido al alto riesgo de sangrado, se decidió iniciar una infusión continua de alteplasa a $1 \mathrm{mg} / \mathrm{h}$ durante $12 \mathrm{~h}$. Posterior al retiro de la sonda EKOS con evidente mejoría hemodinámica. Ecocardiográficamente: VD con función sistólica conservada, TAPSE de 19, onda $\mathrm{S}$ de $10 \mathrm{~cm} / \mathrm{s}$, fracción de acortamiento del $45 \%$; válvula tricúspide con déficit de coaptación que genera jet concéntrico de insuficiencia moderada, PSAP de $50 \mathrm{mmHg}$, proporción VD/VI de 0.78 . A las 48 horas después del TDC se realizó angiografía de control y toma de presiones, y se encontró PSAP $48 \mathrm{mmHg}$, PMAP $30 \mathrm{mmHg}$, y con flujo distal normal en la rama derecha de la arteria pulmonar, sin imagen sugestiva de trombo (Fig. 2 B).

La paciente continuó en internamiento y observación hemodinámica durante 72 horas, dándose de alta asintomática y en clase funcional I del New York Heart Association para seguimiento ambulatorio con anticoagulación a base de rivaroxaban $20 \mathrm{mg}$ al día. A los 2 meses de seguimiento continúa asintomática desde el punto de vista cardiovascular y sin nuevos eventos de embolismo.

\section{Discusión}

Para pacientes sin una contraindicación absoluta para la trombólisis sistémica puede considerarse el TDC, así como se realizó en este caso clínico. El ultrasonido de baja energía desagrega la fibrina dentro de los trombos agudos. Esto es aprovechado por el dispositivo EKOS (BTG-IM, Bothell, Washington), que combina la emisión de ultrasonido de baja energía y la infusión de un agente trombolítico mediante un catéter que contiene múltiples orificios laterales. Esta estrategia se evaluó en el ensayo ULTIMA ${ }^{7}$, que demostró superioridad a la anticoagulación sola para mejorar la hemodinámica sin un aumento significativo de las complicaciones hemorrágicas. El estudio SEATTLE II fue un ensayo multicéntrico de un solo grupo de TDC que demostró una mejoría de los índices hemodinámicos del VD en pacientes con TEP masiva y submasiva ${ }^{6}$. No hubo casos de hemorragia intracraneal; aunque se observaron 16 episodios de sangrado (1 GUSTO grave, 15 GUSTO moderado).

Dada la evidencia disponible del TDC en TEP aguda, el uso de una estrategia de TDC asistida por ultrasonido debe explorarse de forma altamente individualizada. Como se mostró en este caso, los pacientes con TEP aguda demostrada deben ser anticoagulados rápidamente con heparina no fraccionada intravenosa con un bolo de $80 \mathrm{Ul} / \mathrm{kg}$, seguido de una infusión continua ${ }^{10}$. Junto con la evaluación clínica y hemodinámica del paciente, se debe tomar una decisión individualizada con respecto a su idoneidad para la TDC. Una infusión continua de alteplasa a $1 \mathrm{mg} / \mathrm{h}$ en cada arteria pulmonar puede iniciarse mientras se observa al paciente en un entorno de cuidados intensivos ${ }^{8}$. La dosis de alteplasa se puede reducir a la mitad a las 5 horas durante 10 horas adicionales si el riesgo de sangrado del paciente así lo amerita ${ }^{10}$. La dosis máxima recomendada de alteplasa es de $20 \mathrm{mg}$ para la colocación bilateral del dispositivo, y a las 15 horas, como máximo, la infusión de alteplasa debe interrumpirse junto con el sistema de transductor de ultrasonido ${ }^{10}$. El sistema de catéter y la vaina introductora deben retirarse con compresión manual del sitio de acceso hasta que se logre la hemostasia. Se debe realizar un ecocardiograma de seguimiento para confirmar la mejoría en la función del VD.

Se presenta este caso como ejemplo de una aproximación clínica, real y descriptiva del uso de esta modalidad de tratamiento para la TEP aguda. Se trata de un caso con una adecuada evolución clínica y hemodinámica a corto plazo, por lo que se reproduce lo antes planteado de la literatura existente.

EI TDC parece ser una opción viable y segura, con menor riesgo de complicaciones, que se debe utilizar de forma individualizada en casos de pacientes con TEP masiva o submasiva con contraindicaciones para el tratamiento trombolitico o con alto riesgo de hemorragia.

\section{Conflicto de intereses}

Los autores declaran no tener ningún conflicto de intereses.

\section{Financiamiento}

Ninguno. 


\section{Responsabilidades éticas}

Protección de personas y animales. Los autores declaran que para esta investigación no se han realizado experimentos en seres humanos ni en animales.

Confidencialidad de los datos. Los autores declaran que han seguido los protocolos de su centro de trabajo sobre la publicación de datos de pacientes.

Derecho a la privacidad y consentimiento informado. Los autores han obtenido el consentimiento informado de los pacientes y/o sujetos referidos en el artículo. Este documento obra en poder del autor de correspondencia.

\section{Bibliografía}

1. Stephen R, Ken Z, Noor A. Acute Pulmonary Embolism: Endovascular Therapy. Cardiovasc Diagn Ther. 2018; 8(3):244-52.

2. Yamamoto T. Management of Patients with High-Risk Pulmonary Embolism: A Narrative Review. J Intens Care. 2018;6:16.
3. Jimenez D, Yusen RD, Otero R, Uresandi F, Nauffal D, Laserna E. Prognostic Models for Selecting Patients with Acute Pulmonary Embolism for Initial Outpatient Therapy. Chest. 2007;132(1):24-30.

4. Kuo WT, Van den Bosch MA, Hofmann LV, Louie JD, Kothary N, Sze DY. Catheter-directed embolectomy, fragmentation, and thrombolysis for the treatment of massive pulmonary embolism after failure of systemic thrombolysis. Chest. 2008;134:250-4.

5. Konstantinides SV, Barco S, Lankeit M, Meyer G. Management of Pulmonary Embolism an Update. J Am Coll Cardiol. 2016;67(8):976-90.

6. Piazza G, Hohlfelder B, Jaff MR, Ouriel K, Engelhardt TC, Sterling KM et al. A Prospective, Single-Arm, Multicenter Trial of Ultrasound-Facilitated, Catheter-Directed, Low-Dose Fibrinolysis for Acute Massive and Submassive Pulmonary Embolism: The SEATTLE II Study. JACC Cardiovasc Interv. 2015;8(10):1382-92.

7. Kucher N, Boekstegers $P$, Müller O, Kupatt C, Beyer-Westendorf J Heitzer T, el al. Randomized, Controlled Trial of Ultrasound-Assisted Catheter-Directed Thrombolysis for Acute Intermediate-Risk Pulmonary Embolism. Circulation. 2013:129:479-86.

8. Tapson VF, Sterling K, Jones N, Elder M, Tripathy U, Brower J, et al. A Randomized Trial of the Optimum Duration of Acoustic Pulse Thrombolysis Procedure in Acute Intermediate-Risk Pulmonary Embolism: The OPTALYSE PE Trial. JACC Cardiovasc Interv. 2018;11(14):1401-10.

9. Bloomer TL, El-Hayek GE, McDaniel MC, Sandvall BC, Liberman HA, Devireddy CM, et al. Safety of Catheter-Directed Thrombolysis for Massive and Submassive Pulmonary Embolism: Results of a Multicenter Registry and Meta-Analysis. Cath Cardiovasc Interv. 2017;89(4):754-60.

10. Jaber WA, Fong PP, Weisz G, Lattouf O, Jenkins J, Rosenfield K, et al. Acute Pulmonary Embolism: With an Emphasis on an Interventional Approach. J Am Coll Cardiol. 2016;67(8):991-1002. 